\title{
Sal y salud. Poblaciones y territorios Salt and Health. Populations and Territories
}

Elisa Pozo Menéndez, Arquitecta investigadora grupo ABIO UPM

DOI: $10.20868 /$ ciur.2020.128.4393

\section{DESCRIPTORES:}

Sal / Salud / Planificación territorial / Sostenibilidad

\section{KEY WORDS:}

Salt / Health / Territorial Planning / Sustainability

\section{RESUMEN:}

El vínculo entre salud humana y medio ambiente ha adquirido en la actualidad una gran relevancia. Ya en la Antigüedad, las primeras civilizaciones estudiaban los condicionantes del entorno natural para elección de los asentamientos y ciudades. Este conocimiento tradicional se ha ido perdiendo y, muestra de ello, son las enfermedades y epidemias consecuencia de asentamientos inadecuados. En este contexto, la sal se ha utilizado a lo largo de los siglos para usos muy diversos. A medida que la medicina y las investigaciones han ido evolucionando, la sal ha adquirido igualmente una gran visibilidad en cuanto a su relación con la salud. Los hábitos de vida y consumo en la actualidad están perjudicando negativamente no solo a la salud humana, sino a la salubridad medioambiental. La puesta en valor del patrimonio, el paisaje cultural y la artesanía conecta a las personas con los territorios de una forma todavía poco explorada y que requiere mayor investigación. Este trabajo muestra la necesidad de seguir fomentando los estudios interdisciplinares y la colaboración entre distintas áreas de conocimiento, para poder comprender mejor las relaciones causa-efecto entre el medio ambiente, los territorios, hábitos y estilo de vida y la salud humana.

\section{ABSTRACT:}

The connection between human health and environment has recently acquired special relevance. Since antique times, first civilizations already studied environmental conditionants in order to chose the optimal place for settlements and cities. This traditional knowledge has been gradually lost and proof of that are the diseases and epidemies consequence of innacurated settlements. In this context, salt has been used during centuries with different applications. As long as medicine and scientific research have evolved, the salt and its relationship with health have become more visible. Modern lifestyles and consumption patterns are affecting negatively both human health and environmental healthiness. Emphasizing the heritage, cultural landscapes and artisan activities make people develop special connections with territories that need to be explored yet. This paper shows the interest on supporting interdisciplinary studies and collaboration between different fields, in order to better understand the relations cause-effect between environment, territories, habits and lifestyles and human health. 
* Elisa Pozo Menéndez es Arquitecta (Plan 96) especialista en Urbanismo, planificación territorial y medio ambiente por la Universidad Politécnica de Madrid. Máster en Ciudades Sostenibles (Institut Français d'Urbanisme, 2014) y Máster en Medio Ambiente y Arquitectura Bioclimática (Universidad Politécnica de Madrid, 2019). Actualmente investigadora de Doctorado en Sostenibilidad y Regeneración Urbana (Universidad Politécnica de Madrid).

e.pozo@upm.es

ORCID: http://orcid.org/0000-0001-5420-7849 (Elisa Pozo Menéndez)

\section{TERRITORIO Y SALUD}

La relación entre la salud humana y los factores ambientales ha adquirido gran importancia en la actualidad. Para la promoción de la salud como objetivo prioritario del siglo XXI, la Organización Mundial de la Salud lanzó una campaña en 2010 para dar visibilidad a la necesidad de una planificación urbana centrada en la salud de la población (OMS, 2010). Tanto en los países desarrollados como en los que están en desarrollo, el foco de atención está puesto en el entorno urbano debido a que más de la mitad de la población mundial habita en ciudades y áreas metropolitanas. Sin embargo, los ecosistemas territoriales son un conjunto de relaciones complejo y es necesario pensar en un equilibrio entre los sistemas rurales y urbanos para favorecer ambientes saludables y sostenibles en todas las escalas (ONU Habitat, 2012, 2015; FAO, 2018).

Esta preocupación por el medio ambiente en relación con la salud de las personas no es nueva. Ya las primeras civilizaciones mediterráneas incluían unas reglas de planificación territorial para el asentamiento de las ciudades donde la localización de las mismas era clave para la salud de sus habitantes y para su prosperidad. Por ejemplo, la mayoría de las ciudades etruscas se ubicaban en zonas elevadas, donde poder observar todo el terreno circundante. Pero además, la decisión de elegir un lugar u otro tenía que ver con los condicionantes del territorio. Muchas de estas ciudades se asentaron en suelos muy fértiles, de gran riqueza mineral, lo cual se tradujo en la prosperidad de las actuales ciudades italianas como Felsina (Bolonia), Faesole (Fiesole), Arrétion (Arezzo) o Velzna (Orvieto) (Walker J.M., 2003).

Estas normas fundacionales etruscas se adoptaron por los romanos. La leyenda de Rómulo y Remo sobre la fundación de Roma detalla cómo la observación del vuelo de las aves fue uno de los condicionantes para la decisión de su ubicación y el motivo del asesinato de Remo a manos de su hermano. Los ritos fundacionales tenían una gran carga religiosa: los augures (sacerdotes) examinaban las entrañas de las aves y de otros animales cazados en las inmediaciones del lugar en un altar erigido en el futuro centro de la ciudad. La interpretación de los augures de lo que veían en las vísceras de los animales determinaba el futuro de la ciudad y, si los signos eran favorables, la ciudad podía fundarse. Este rito, marcado por las interpretaciones de los poderes religiosos, en realidad estaba estrechamente vinculado con la "ciencia" de fundar ciudades y tenía una explicación racional. 


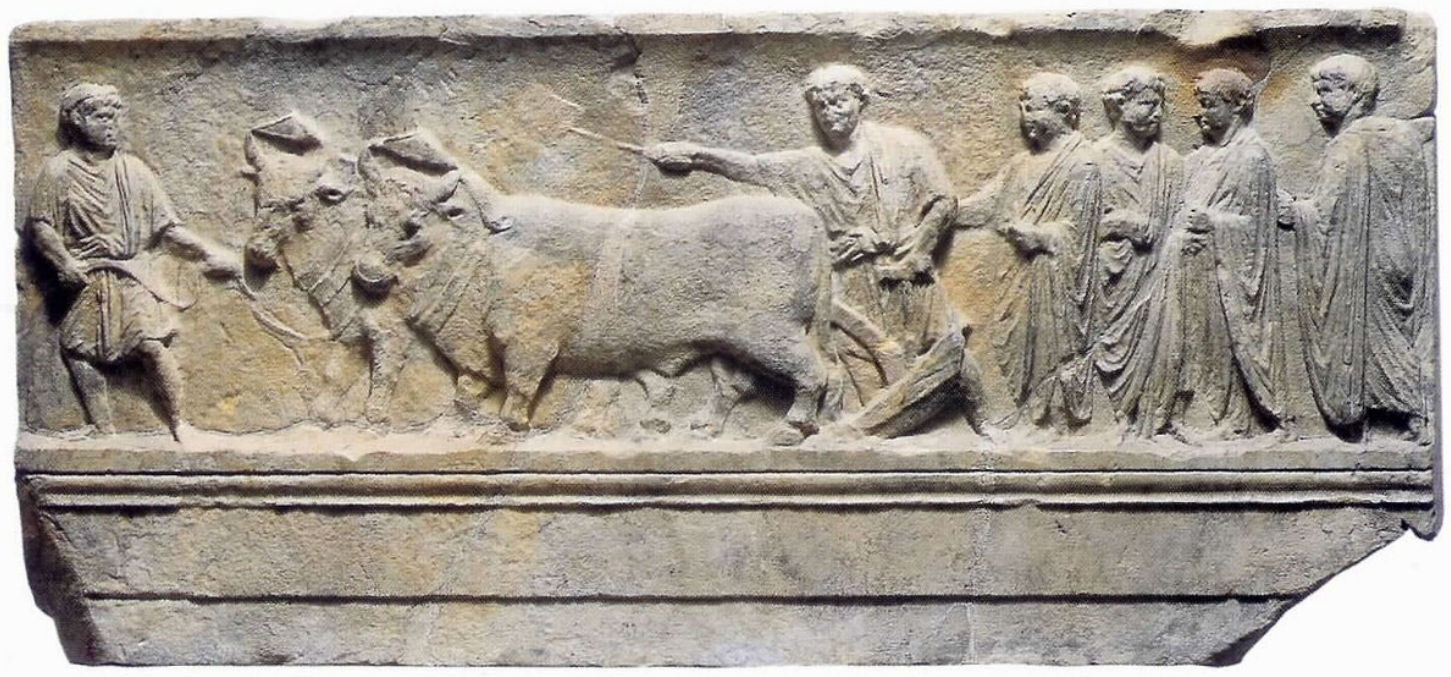

Figura 44. Relieve del Museo Arqueológico de Aquileia (Italia) donde se representa el ritual sagrado de fundación de una ciudad con el arado para delimitar con el surco el perímetro elegido. Fuente: Twitter Antigua Roma al Día. @antigua_roma. Publicado el 21/04/2017. Consultado el 11/01/2020.

Este componente religioso, como sucede con otras muchas costumbres, convenciones y normas, estaba estrechamente vinculado a la ciencia de fundar ciudades y tenía una explicación racional. Vitruvio describe "... al inmolar animales que habían estado pastando en parajes donde se levantaban fortalezas o campamentos fijos, examinaban sus hígados y si los encontraban amoratados y enfermos, inmolaban otros animales ante la duda de si estaban enfermos por alguna indisposición, o bien por tomar pastos en mal estado.Como eran muy expertos, cuando veían que los hígados estaban sanos, por alimentarse de agua $y$ de pastos, precisamente en ese lugar levantaban sus fortificaciones" (Vitruvio Polion, M.L., Los diez libros de arquitectura, Capítulo 4, Libro Octavo).

También Felipe II en las leyes de Yndias de 1573 recogía las indicaciones para la fundación de las ciudades coloniales: "...elijasse la prouincia comarca y tierra que se a de poblar teniendo consideraçion a que sean saludables lo qual se conocera en la copa que huviere de ombres viejos y moços de buena complision dispusicion y color y sin enfermedades $y$, en la copia de animales sanos y de competente tamaño. $Y$ de sanos frutos y mantenimientos, que no se críen cosas ponçonossas y noçibas, de buena y felice costelaçion el çielo claro y begnino el ayre y suaue sin impedimento ni alteraciones..." (Morales Padrón F., 1979). 


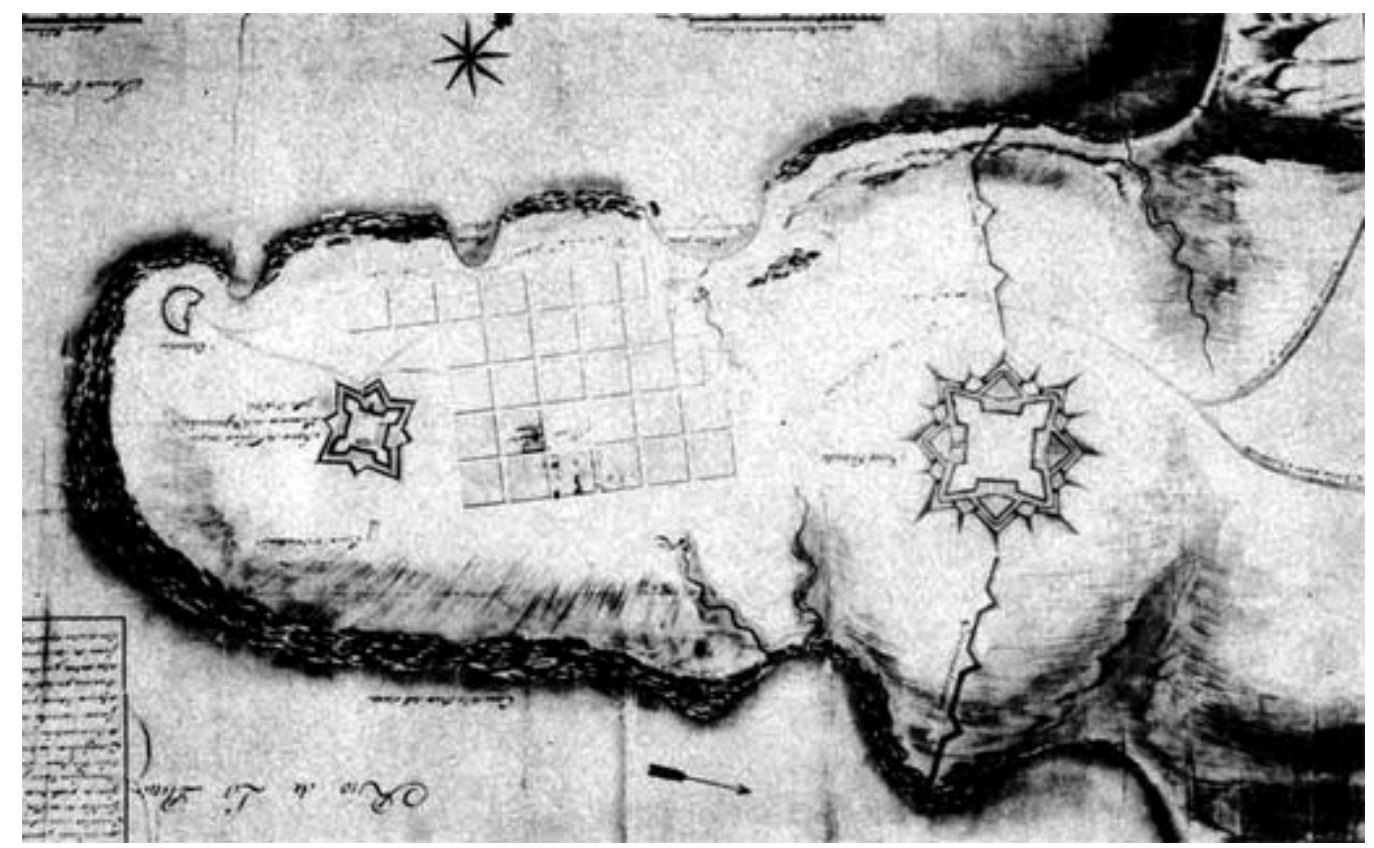

Figura 45. Plano de Montevideo dibujado por Domingo Petrarca alrededor de 1730. A pesar de que la Ciudadela finalmente se construyó más al este en 1741, se mantuvo la disposición original del trazado del damero.

Fuente: Urban Networks (2014). Tres plazas en el Montevideo Antiguo: la sumisa, la rebelde y la conciliadora. Disponible en: http://urban-networks.blogspot.com/ Publicado el 18/10/2014. Consultado el 11/01/2020.

Estas leyes también incluían una previsión que en la zona hubiera recursos naturales disponibles, como ríos y bosques, para poder abastecerse de agua para el consumo y riego, alimentos y materiales de construcción, entre otros. Cabe destacar también cómo en estos artículos se incluían recomendaciones para que estos recursos y las tierras de cultivo estuviesen en áreas circundantes a las nuevas colonias, resultando en economías locales y un consumo de proximidad. Todos estos artículos tenían como objetivo establecer una serie de medidas para la salud y seguridad de sus habitantes, así como el desarrollo y la prosperidad de la colonia, ya que muchas de las que fueron fundadas en las primeras expediciones tuvieron que ser abandonadas por no reunir unas condiciones favorables para vivir en ellas (Sánchez López, 2007).

Estas reglas fundacionales, presentes en muchas culturas y que a día de hoy podríamos identificar con muchos de los criterios bioclimáticos y elementos de la arquitectura tradicional o vernácula (Neila González, 2004), se han ido perdiendo progresivamente. Las necesidades históricas de las poblaciones y asentamientos, los cambios demográficos de las sociedades y la evolución de la tecnología junto con los sistemas de producción, así como de construcción, han permitido al ser humano adaptar su hábitat, las ciudades, gracias a los avances tecnológicos y técnicos. Sin embargo, los nuevos emplazamientos no siempre son los más adecuados. 


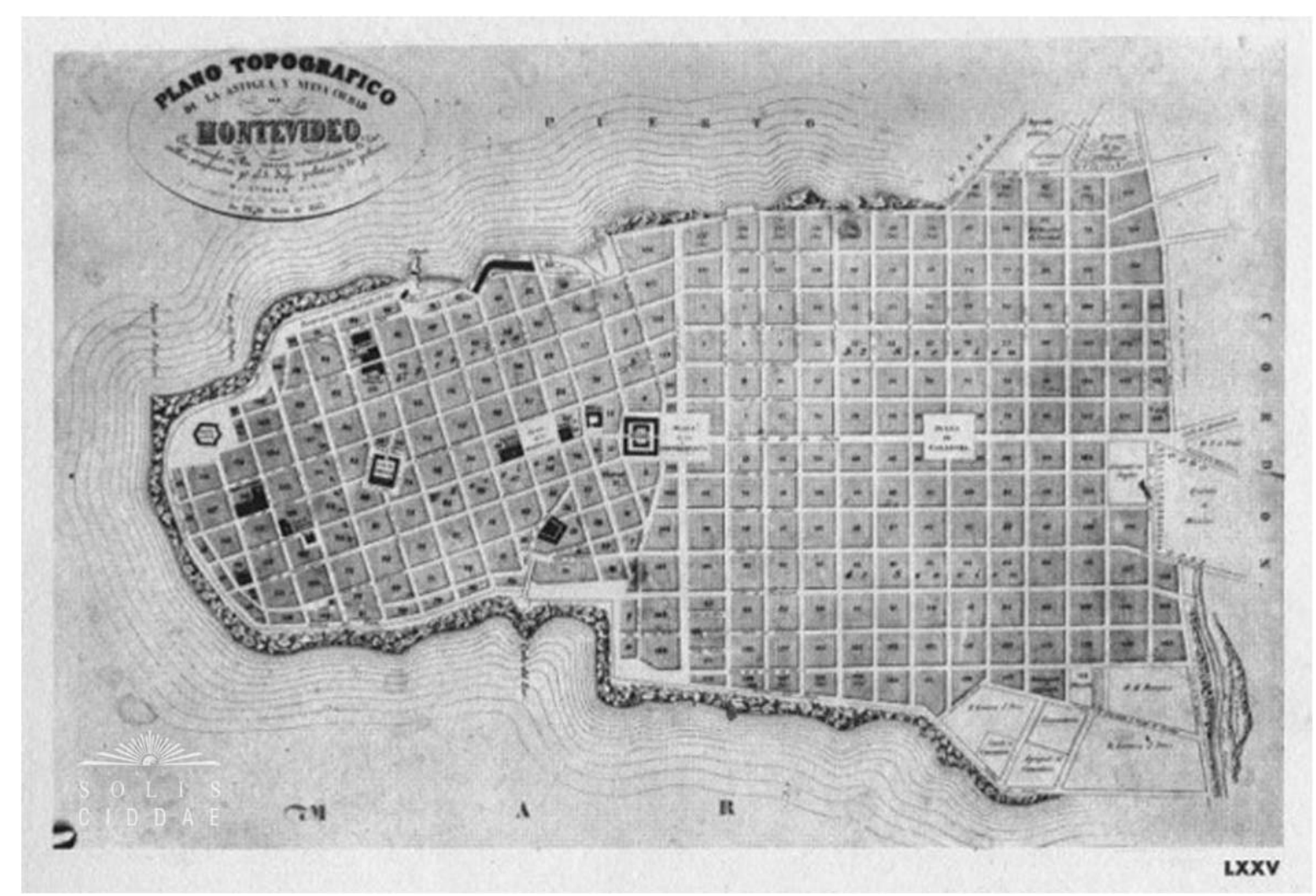

Figura 46. Plano de Montevideo de 1843. Se destaca la expansión de la ciudad hacia el este mantiendo la geometría del trazado del damero pero cambiando su orientación. Se desconoce si la retícula está adaptada a los criterios bioclimáticos más adecuados (viento y soleamiento).

Fuente: Homo Artis (2010). La arquitectura neoclásica en el Uruguay. Disponible en: http://homo-

artis.blogspot.com/2010/05/la-arquitectura-neoclasica-en-el.html. Publicado el 08/05/2010. Consultado el 11/01/2020.

Aunque las metodologías de planificación territorial y las normativas urbanas actuales proponen unas regulaciones sobre usos recomendables y óptimos del suelo y la localización de distintas actividades, sigue siendo un reto el control de los desarrollos urbanos respetando estos principios. Además, todavía existen países y regiones que no cuentan con los estudios territoriales e información adecuada que permitan una propuesta saludable de planificación territorial. La importancia del urbanismo y la planificación territorial para asegurar la salud de la población es clave. La disciplina del planeamiento requiere aportaciones de varios campos y sectores. La evolución de la medicina y de las investigaciones asociadas a la salud, nutrición, psicología y epidemiología tienen una estrecha relación con la geología, geografía, arquitectura e ingeniería. Un marco holístico interdisciplinar que es necesario continuar desarrollando para poder comprender mejor las relaciones causa efecto de los factores ambientales en la salud de las personas.

Por lo tanto, si pensamos en los factores ambientales de las ciudades y los hábitos de consumo, también es necesario un enfoque multidisciplinar que permita establecer unas relaciones y otras para la predicción y prevención de la salud.

A continuación se presenta el caso de la sal y cómo este elemento, indispensable para la vida, interactúa con el ser humano en todas las escalas. 


\section{SAL, EQUILIBRIO Y SALUD}

La sal es una sustancia compuesta de sodio y cloro, macronutrientes imprescindibles no solo para la vida humana, sino para todos los organismos animales y vegetales. Como la mayoría de las sustancias presentes en nuestro organismo, la dosis correcta de cada una de ellas asegura la diferencia entre un veneno y un remedio (Paracelsus, 1493-1541) o estado natural (Bressan, 2014). Así pues, para asegurar una buena salud y bienestar para las personas, se necesita la regulación de la homeostasis de la sangre. Es decir, un equilibrio entre el agua, sodio y otros tantos minerales y sustancias que se necesitan para el correcto funcionamiento de nuestro organismo y sus funciones.

Las recomendaciones de la Organización Mundial de la Salud y otras instituciones en cuestiones de hábitos de vida van variando a lo largo de las décadas y se van adaptando a los nuevos retos y problemas de salud que van apareciendo en la sociedad, así como a las evidencias científicas que se van demostrando. Por ejemplo, las recomendaciones asociadas al consumo de sal hace años se basaban en la supuesta pérdida de los niveles de yodo de este oligoelemento desde su obtención hasta su consumo. Así, la cantidad recomendada era entre 5 y $10 \mathrm{~g} /$ persona/día, considerando una pérdida entre el $25 \%$ y el $50 \%$ de yodo. En la actualidad, aunque los problemas relacionados con el déficit de yodo se han reducido significativamente sobre todo en los países desarrollados, otros problemas han aparecido como la hipertensión 0 el hipertiroidismo (OMS, ICCIDD y UNICEF, 1996).

A día de hoy, la misma Organización Mundial de la Salud recomienda un consumo de sal de $5 \mathrm{~g} /$ persona/día, lo que supone un consumo de sodio de 2 g/día (OMS, 2012). El objetivo es la reducción del riesgo de enfermedades cardiovasculares, accidentes cerebrovasculares y cardiopatía coronaria en adultos. La presencia de sal en el agua y en alimentos que consumimos hace que esta cantidad en realidad sea menor. Existen también recomendaciones que indican que una reducción hasta los $3 \mathrm{~g} /$ persona/día tendrían un mayor efecto en la reducción de la presión arterial (He, Li y MacGregor, 2013).

Sin embargo, en estas cifras la OMS también incluye el sodio de otras fuentes alimentarias distintas de la sal, como el glutamato monosódico y los conservantes (OMS, 2003), que en la actualidad están generando mucha controversia sobre su consumo. También se hace mención al uso de sucedáneos enriquecidos en potasio y pobres en sodio para poder ayudar a la reducción de la ingesta de sodio en muchas dietas. La sal que consumimos se puede dividir en "sal visible", aquella que utilizamos para condimentar los platos (alrededor de un $20 \%$ del consumo total de sal) y la "invisible", que constituye la que procede de los alimentos ( $80 \%$ del consumo total de sal). Esta "sal invisible" al mismo tiempo se divide en la que está presente de forma natural en los alimentos (8$10 \%$ del consumo de sal total) y la que se añade a los alimentos procesados (70$72 \%$ del consumo de sal total) (Cancer Minchot E., y Botella Romero F., 2019).

A pesar de los problemas que se asocian a un consumo de sal excesivo, como es la hipertensión, es necesario recordar que los minerales que la sal aporta son indispensables para una buena salud y no es conveniente prescindir de ella en la dieta, si no incorporarla de una forma equilibrada. 


\subsection{Consumo y nutrición}

Los cambios en la alimentación y una mejor nutrición han sido algunos de los condicionantes que han favorecido la longevidad del ser humano y su calidad de vida. Sin embargo, la ingesta de alimentos no siempre está asociada a un aporte suficiente de los nutrientes que el ser humano necesita para un correcto desarrollo. En este sentido, el ejemplo del yodo nos permite entender la interrelación entre salud, territorio y poblaciones. El yodo es un micronutriente fundamental en la dieta de los seres vertebrados para la producción de hormonas tiroideas. Su déficit está asociado con diversas anomalías funcionales, metabólicas y problemas de desarrollo. Un déficit de yodo durante el embarazo puede tener un impacto en el desarrollo fetal como causa de discapacidad física e intelectual así como cretinismo. El yodo se ingiere a través del pescado blanco y los lácteos, principalmente, pero también de la sal marina.

Sin embargo, no todos los alimentos contienen el mismo porcentaje de micronutrientes. Los animales precisan también de una ingesta de sal de forma regular, actualmente incorporada en los piensos comerciales o a través de bolas o bloques de sal en ganadería de pasto. En el caso de los lácteos, por ejemplo, el ganado necesita sal y yodo pero en algunas regiones, la vegetación que sirve de alimento y pasto no contiene las cantidades necesarias. En zonas que han sufrido fuertes procesos de meteorización y lixiviado por precipitaciones y nieve o zonas afectadas por glaciaciones, normalmente regiones intracontinentales, existe una menor concentración de yodo en las capas superficiales de la tierra y, por tanto, existe un déficit que puede tener consecuencias muy serias en la salud de la población (Izquierdo M., 2018).

La disponibilidad y acceso a alimentos con valor nutricional, tanto físico como social y económico, constituye uno de los desafíos globales reconocido por las Naciones Unidas como objetivo de desarrollo sostenible para el 2030 (ONU, 2015).

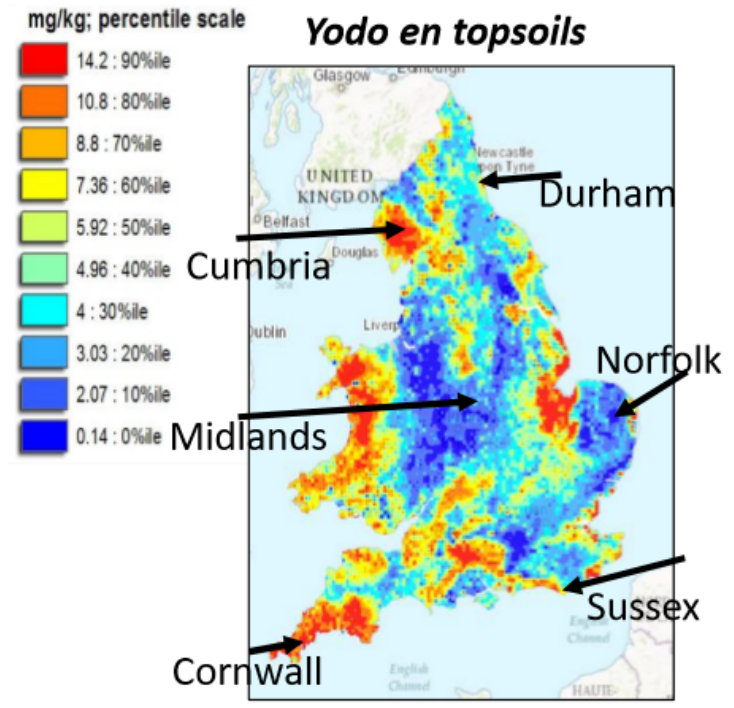

Figura 47. Concentración de yodo en las capas superficiales del suelo. Plano de Ingraterra y Gales. Fuente: Izquierdo M. (2018). Hambre Oculta. Déficit de micronutrientes y enfermedades asociadas en I Curso de Especialización en Geología Médica Salamanca. 
Gracias a su origen natural, la sal ha sido siempre un aditivo seguro en la alimantación humana. La sal yodada o las sales enriquecidas con flúor u otros minerales han sido incorporadas en la dieta de muchas poblaciones y países como un complemento necesario para asegurar un desarrollo saludable de la población. La puesta en valor de la sal como producto y su calidad en el proceso de elaboración de los alimentos tiene por tanto un especial interés para asegurar el equilibrio en la dieta de la población tanto urbana como rural. De la misma forma en que los humanos complementamos la alimentación con más o menos sal para un correcto funcionamiento, el ganado requiere también un complemento de sal para una producción de leche de calidad o para reponer minerales de grandes esfuerzos de trabajo (Menéndez Pérez, 2008).

Continuando con la evidencia de que los entornos urbanos influyen en la salud de la población, el acceso a productos de calidad en el barrio donde vivimos también condiciona en cierta medida nuestros hábitos de consumo y alimentación (Cebrecos, Díez, Gullón et al., 2016). En las grandes ciudades se pone de relieve cada vez más la búsqueda de alternativas a lo que se ha asociado a los sistemas urbanos en todos los aspectos. La aparición de huertos urbanos y cooperativas de consumo es uno de los ejemplos más estudiados a nivel urbano y que están sacando a la luz una demanda por nuevas formas de consumo y una mayor preocupación por un estilo de vida saludable, donde se pone de valor la alimentación en primer lugar, pero también el contacto con la naturaleza, los productores locales y el fortalecimiento de las redes de proximidad en los barrios. En definitiva, la búsqueda de unos ritmos y una forma de entender los procesos humanos desde la alimentación hasta la forma de trabajar en consonancia con nuestra salud en todos los aspectos (Llobera Serra, 2014).

\subsection{Otras aplicaciones y usos}

Además de su utilización como condimento en la alimentación, la sal tiene gran importancia en muchas otras aplicaciones antrópicas. Durante siglos, la sal ha sido utilizada en la conservación de alimentos. Gracias a este uso de la sal se hizo posible el transporte y almacenamiento de provisiones y alimentos durante períodos más largos de tiempo. Este hecho permitió el desarrollo de la ganadería y pesca, favoreciendo el paso del estilo nómada al sedentario.

Por otra parte, las primeras referencias escritas sobre la producción de la sal están recogidas en el Pen tzao Kang-mu (2679 a.C.), un tratado chino donde se detallan más de cuarenta tipos de sal, sus propiedades y las diferentes formas de obtención y utilización para su consumo. Sin embargo, es a partir del siglo XIX donde la sal adquiere mayor interés en distintos procesos industriales, como la industria peletera, la química o la farmacéutica. En el caso de la industria química, la sal se utiliza para el mantenimiento y esterilización del agua en piscinas, por ejemplo. Asímismo, también se destaca su uso para el mantenimiento de carreteras en los meses de invierno. Así, el 91\% de la producción de la sal se destina al consumo humano, a la industria química y al mantenimiento de viario (Román López E., 2014).

A pesar de que el uso de la sal en carretera tiene algunos inconvenientes, resulta uno de los anticongelantes más económicos y naturales para el 
mantenimiento del viario en condiciones extremas de invierno. Los problemas derivados de su impacto en la naturaleza secando la hierba y árboles y afectando a la alimentación de algunos animales pueden limitarse utilizando la sal en proporciones adecuadas y confinando al máximo su aplicación sobre el viario estrictamente.

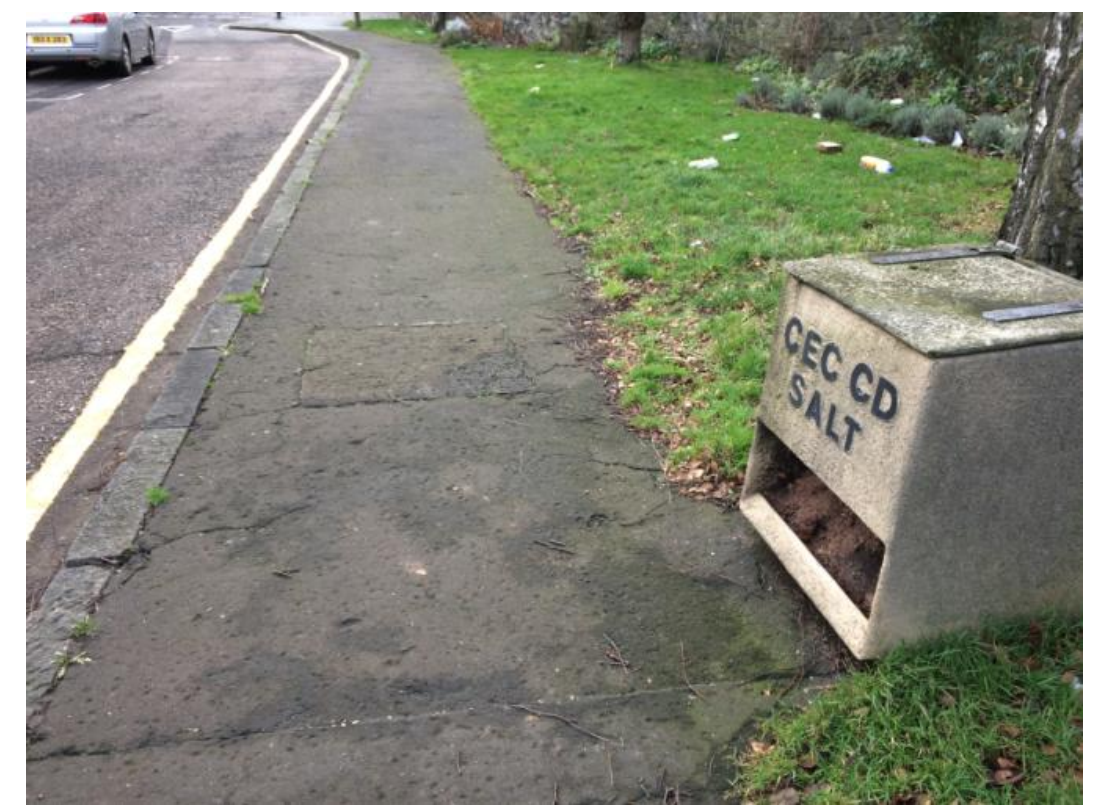

Figura 48. Contenedor de sal como parte del mobiliario y servicios urbanos en las calles de Edimburgo (Escocia).

Fuente: Pozo Menéndez, E. (2019).

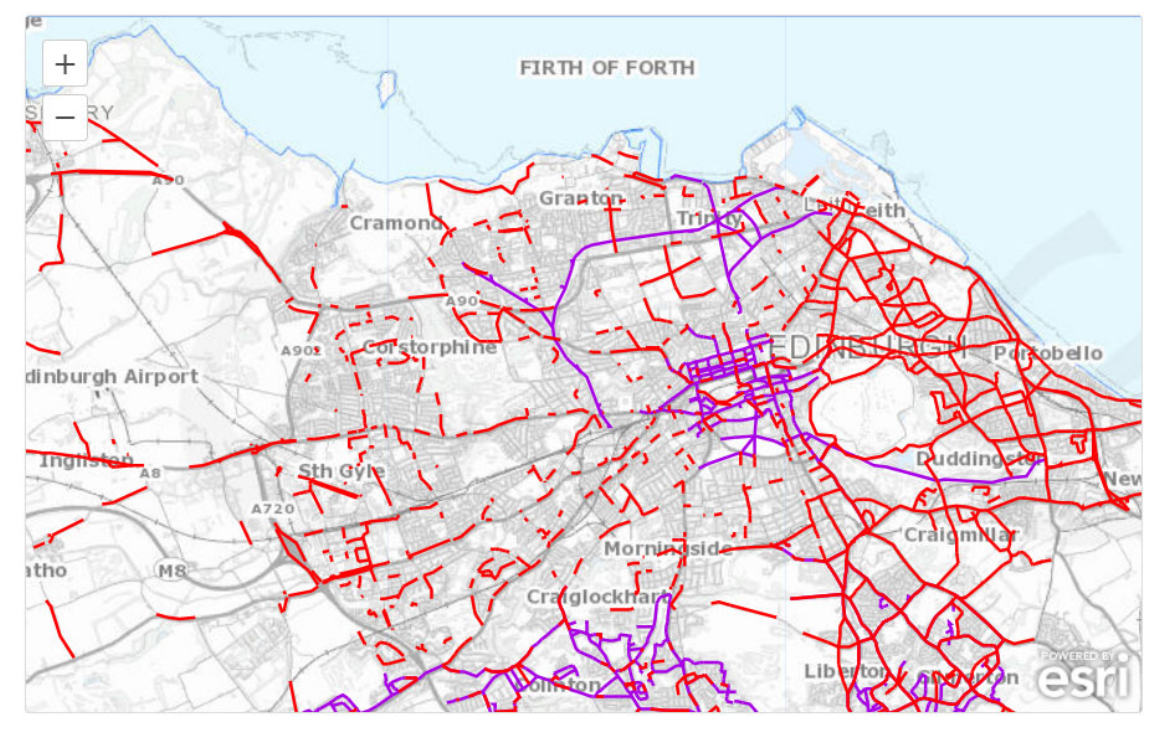

Figura 49. Plano de rutas prioritarias para quitanieves con sal y contenedores de sal en Edimburgo (Escocia). En rojo, los viarios de máxima prioridad y en morado, los viarios de prioridad secundaria, según las condiciones meteorológicas.

Fuente: Ayuntamiento de Edimburgo, Escocia. Disponible en: https://www.edinburgh.gov.uk/ Consultado el 11/01/2020.

Por lo tanto, la producción de sal resulta de gran relevancia más allá de la preservación del paisaje y del patrimonio cultural asociados a las explotaciones 
salineras artesanas. La sal permite igualmente mantener y conservar la biodiversidad existente en estos territorios $y$, para muchas comunidades, establece una forma de vida que establece una simbiosis con la naturaleza, explotando los recursos naturales en un equilibrio deseable para la sostenibilidad en todos los aspectos. En este sentido, también es necesario considerar los beneficios de los recursos naturales sobre la salud, incluyendo los servicios ecosistémicos que éstos nos ofrecen (FAO, 2015).

\subsection{Efectos terapéuticos de la sal}

La sal y el agua marina han sido utilizadas a lo largo de la historia por sus propiedades terapéuticas y sanadoras. Desde la Antigüedad, la sal se incorporó en la Medicina como remedio de diversos síntomas y enfermedades. Los baños de agua con sal o la aplicación de plantas y sustancias del mar Rojo y del mar Mediterráneo son algunos de los ejemplos de remedios con sal recogidos en tratados, papiros y otros documentos escritos. La sal se ha empleado como remedio en distintas culturas, desde Mesopotamia hasta Grecia y el mundo árabe, para paliar dolores y enfermedades, así como intoxicaciones o picaduras de algunos animales e insectos.

En la actualidad, la sal sigue estando presente en los remedios caseros y fórmulas farmacéuticas en diversos formatos. Se puede encontrar como desinfectante aplicado por vía tópica, es un antihistamínico natural y también se utiliza como remedio para problemas respiratorios como sinusitis o congestión nasal mediante lavados o inhalaciones de agua salada. Asimismo, durante siglos la sal se ha utilizado mezclada con otras sustancias creando ungüentos, cataplasmas y mejunjes diversos para tratamientos de salud, bienestar y belleza. Se utiliza como tratamiento contra la gota, sabañones, artritis, artrosis y problemas cutáneos como la psoriasis, acné o dermatitis atópica. Esta mezcla de la sal con otras sustancias como miel, vinagre o aceite, se han utilizado como remedios bastante eficaces basados en la observación empírica (Porres Marijuán, 2003).

También el agua salada como remedio medicinal ha sido utilizada en balnearios y spas desde el siglo XIX para tratar problemas musculares, enfermedades de la piel o como estancias para combatir fatiga, estrés, cansancio y problemas de insomnio. En la literatura, podemos encontrar citas de Palacio Valdés alabando los baños de mar y la brisa salina a través de varias obras (Girón Irueste, 2006). Actualmente, en las ciudades han aparecido igualmente balnearios y centros de haloterapia para tratamientos alternativos y prevención de enfermedades, de los que aún existen escasas evidencias científicas sobre sus beneficios en la salud de las personas, pero es una práctica cada vez más extendida.

\section{URBANISMO CENTRADO EN LAS PERSONAS}

A día de hoy, los grandes retos de la humanidad ponen el foco de atención en el contexto urbano por ser el hábitat de más de la mitad de la población mundial. El cambio climático, la transformación de la estructura demográfica tradicional y la salud en las ciudades son algunos de los temas que más preocupación generan y constituyen los principales retos actuales (OMS, 2010). De la misma forma en 
que los servicios de salud están evolucionando hacia modelos centrados en la persona, con este trabajo se propone un enfoque análogo para la práctica de la arquitectura, urbanismo y planeamiento territorial. Las ciudades, como hábitats propios de los seres humanos, caracterizados por el intercambio social, de bienes, mercancías, productos, cultura, materia y energía, entre otros factores, podrían transformarse con el acento en la salud de la población y la prevención. Esta visión, se complementa con los critesis de urbanismo sostenible en los que se lleva investigando durante tantas décadas.

En el ser humano hablamos de homeostasis como aquella capacidad de los organismos para mantener unas condiciones internas estables a través del intercambio regulado de materia y energía para compensar los cambios en su entorno. De la misma forma, las ciudades y asentamientos humanos pueden comportarse de forma similar con su entorno próximo mediante sistemas complejos que permitan ciclos circulares de energía y materia.

La conexión de las ciudades con las prácticas tradicionales, el mundo rural y la producción de los alimentos desde su extracción puede ser una de las estrategias necesarias para asegurar la buena salud de la población urbana y una mayor concienciación sobre unos hábitos de alimentación saludables. En este aspecto, se precisa mayor investigación para poder dilucidar con precisión las relaciones causa efecto entre factores ambientales, de consumo y la aparición de enfermedades. Algunas ideas para futuras líneas de investigación que podrían estudiarse explorando este enfoque multidisciplinar serían:

- Reconocer la influencia del entorno de las salinas en la salud humana y comprender las vías por las que las personas entran en contacto con él: suelo, agua, aire o polvo.

- Agricultura urbana y periurbana y trazabilidad de micronutrientes.

- Estudios sobre prevención y salud de trabajadores en los entornos productivos de las salinas.

- Calidad del agua de abastecimiento de las diferentes localidades europeas y estudios epidemiológicos urbanos. Guía de recomendaciones según localidades y propiedades minerales de sus aguas locales.

- Nuevas aplicaciones de la sal en proceso y fabricación de materiales de diseño y construcción.

Los problemas de salud asociados a las ciudades de la sociedad moderna como la obesidad y los problemas cardiovasculares, las enfermedades pulmonares y respiratorias o la salud mental, entre otros (NHS London Healthy Urban Development Unit, 2007) son la consecuencia del desarrollo de un sistema que aunque viene asociado a un gran progreso de la humanidad, se ha mostrado deficiente en asegurar un bienestar real de la población. El presente trabajo incide en la creciente necesidad en adaptar los territorios y los flujos y dinámicas de las ciudades a los retos de sostenibilidad y de salud que la sociedad actual demanda. 


\section{BIBLIOGRAFÍA}

Bressan D. (2014). Physician Paracelsus and early Medical Geology. History of Geology. Blog Scientific American. Disponible en: https://blogs.scientificamerican.com/history-of-geology/physician-paracelsusand-early-medical-geology/

Cancer Minchot E., y Botella Romero F. (2019). La sal en nuestra alimentación. Sociedad Española de Endocrinología y Nutrición SEEN.

Cebrecos, A., Díez, J., Gullón, P. et al. (2016). Characterizing physical activity and food urban environments: a GIS-based multicomponent proposal. International Journal of Health Geographics. 15:35. DOI:10.1186/s12942-0160065-5

FAO (2018). Ecosystem Services \& Biodiversity (ESB). Disponible en: http://www.fao.org/ecosystemservicesbiodiversity/resources/en/

Fariña J., Higueras E., Román E. (2018). Ciudad, urbanismo y salud. Criterios generales de diseño urbano para alcanzar los objetivos de una ciudad saludable. Envejecimiento activo.

Girón Irueste F. (2006). Uso médico del agua en el mundo hispánico bajo medieval. Balnea No. 1, pp. 79-95.

He F.J., Li J. MacGregor G.A. (2013). Effect of longer-term modest salt reduction on blood pressure. Cochrane Systematic Review - Intervention. Disponible en: https://doi.org/10.1002/14651858.CD004937.pub2

Homo Artis (2010). La arquitectura neoclásica en el Uruguay. Disponible en: http://homo-artis.blogspot.com/2010/05/la-arquitectura-neoclasica-en-el.html. Publicado el 08/05/2010. Consultado el 11/01/2020.

Izquierdo M. (2018). Hambre Oculta. Déficit de micronutrientes y enfermedades asociadas en I Curso de Especialización en Geología Médica Salamanca. Celebrado el 11/11/2018.

Llobera Serra P. (2014). Horticultura urbana: La Red de Huertos Urbanos Comunitarios de Madrid. Ambienta. No. 107. Pp. 120 - 128.

Menéndez Pérez, E., 2008. Las rutas de la sal. A Coruña: NETBIBLO, S.L.

Morales Padrón, F. (1979). Ordenanzas de Felipe II sobre descubrimiento, nueva población y pacificación de las Indias en Teoría y leyes de la conquista. Ediciones Cultura Hispánica del Centro Iberoamericano de Cooperación, Madrid, pp. 489518.

NHS London Healthy Urban Development Unit (2007). Delivering Healthier Communities in London. Land use consultants in association with the centre for research into environment and health. 
Neila González J. (2004). Arquitectura bioclimática en un entorno sostenible. Ed. Madrid: Munillairea.

OMS, ICCIDD, UNICEF (1996). Recommended iodine levels in salt and guidelines for monitoring their adequacy and effectiveness. Disponible en:

https://www.who.int/nutrition/publications/micronutrients/iodine_deficiency/WH O_NUT_96.13/en/

OMS (2010). La planificación urbana es esencial para la salud pública. Comunicado de prensa del Día Mundial de la Salud. Disponible en: https://www.who.int/mediacentre/news/releases/2010/urban_health_20100407/ es/

OMS (2013). Ingesta de sodio en adultos y niños. Departamento de Nutrición para la Salud y el Desarrollo. Organización Mundial de la Salud.

ONU Habitat (2012). Estado de las Ciudades de América Latina y el Caribe.

Rumbo a una nueva transición urbana. Programa de las Naciones Unidas para los Asentamientos Humanos.

ONU (2015). Objetivos y metas de desarrollo sostenible. Disponible en: https://www.un.org/ sustainabledevelopment/es/objetivos-de-desarrollosostenible/

Porres Marijuán, R., 2003. Sazón de manjares y desazón de contribuyentes. La sal en la Corona de Castilla en tiempos de los Austrias, Zarautz (Guipuzkoa): Universidad del País Vasco.

Román López E. (2014). Paisajes de la sal en Andalucía. (Tesis doctoral). Universidad Politécnica de Madrid.

Sánchez López (2007). La ciudad en el Nuevo Mundo según las Ordenanzas de 1573. Asociación Cultural Coloquios históricos de Extremadura. Disponible en: https://chdetrujillo.com/la-ciudad-en-el-nuevo-mundo-segun-las-ordenanzas-de$1573 /$

Urban Networks (2014). Tres plazas en el Montevideo Antiguo: la sumisa, la rebelde y la conciliadora. Disponible en: http://urban-networks.blogspot.com/ Publicado el 18/10/2014. Consultado el 11/01/2020.

Vitruvio Polion, M.L., Los diez libros de arquitectura.

Walker J.M. (2003). Los etruscos. Madrid: Edimat. 